\title{
Ocular pathology associated with the Weber Christian syndrome
}

\author{
J. FREEDMAN
}

Department of Ophthalmology, University of the Witwatersrand, and Johannesburg General Hospital, South Africa

Anterior and posterior uveitis has been described as an associated feature of the Weber Christian syndrome (Klien, I959), but only one report of a case has thus far appeared in the literature. The presence of uveitis in sections of an eye removed from a patient suffering from Weber Christian disease was described by Theobald (I953).

Frayer, Wise, and Tsaltas (I968) described three cases of Weber Christian syndrome with associated ocular features: one patient had a small tender nodule on one eyelid, another had subconjunctival nodules next to the limbus, and the third showed a yellow limbal mass with episcleral and conjunctival congestion. Thus these three cases all showed anterior segment involvement and no uveal tract lesions.

The occurrence of bilateral retinal vascular abnormalities and macular haemorrhages in association with the Weber Christian syndrome does not appear to have been reported in the literature.

\section{Case report}

A 62-year-old woman came to the Eye Clinic of the Johannesburg General Hospital with the complaint of a sudden diminution of vision in both eyes, and a "central black area" when reading.

She volunteered the information that she was a sufferer from Weber Christian syndrome. The disease, which was still active, manifested itself in the form of recurrent crops of painful nodules on the under surfaces of the thighs. The diagnosis had been proved by histological examination of a biopsy specimen of a nodule, which showed areas of congestion, chronic cell infiltrate, and foci of fat necrosis. Apart from Weber Christian disease, she did not suffer from hypertension, diabetes, or rheumatoid arthritis.

\section{Examination}

At the first visit the visual acuity was $6 / 60$ in both eyes, with full correction. The ocular movements were normal and the anterior chambers and vitreous free of flare and cells. At each macula there were almost identical oval-shaped haemorrhages. The retinal arterial vessels in the region of the macula were tortuous and showed looping and atypical cork-screw formation. The veins appeared to be normal (Figs I and 2).

Fluorescein angiography showed the presence of a leak at both maculae.

\section{Laboratory investigations}

Erythrocytes 4. 15 millions; haemoglobin $13 \mathrm{~g}$. per cent; white cell count 3,90o, with normal differential count; erythrocyte sedimentation rate $7 \mathrm{~mm}$. Ist hr. Total lipid $639 \mathrm{mg}$./per cent.; total cholesterol $188 \mathrm{mg}$./per cent.; triglycerides I I $\mathrm{mg}$./per cent. Fasting blood sugar $87 \mathrm{mg}$./per cent., total protein $6.4 \mathrm{mg}$./per cent., Albumin 58.9 per cent., alpha I, II, beta, and gamma globulins all normal. Cryglobulins absent. 


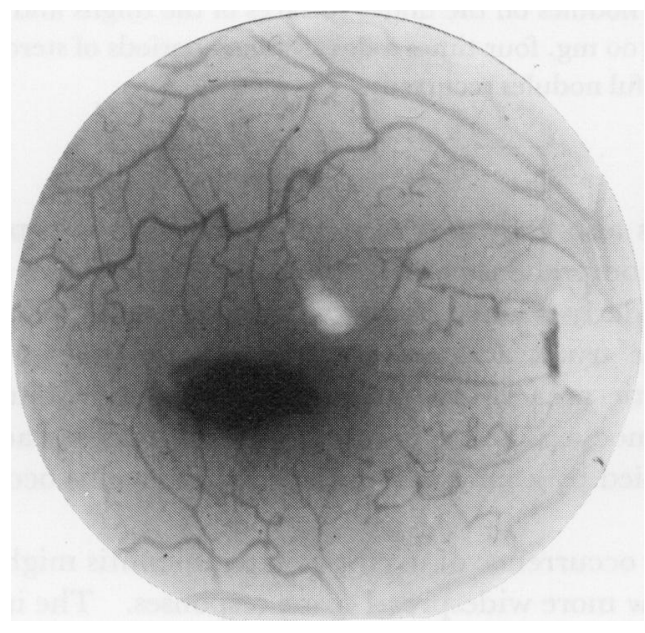

FIG. I Right eye at first examination

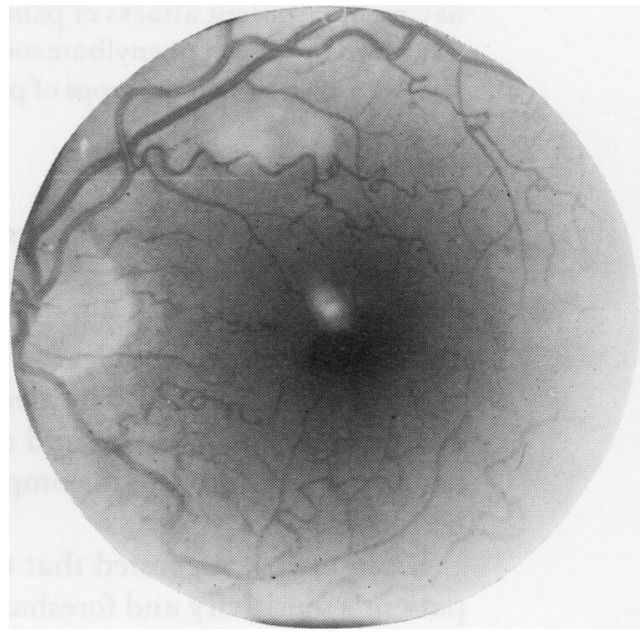

FIG. 2 Left eye at first examination

\section{Treatment}

No therapy was given for the ocular lesions and the patient was seen again 2 weeks later, when the haemorrhages had absorbed to a large extent and the vision had returned to $6 / 9$ both eyes (Figs 3 and 4 ).

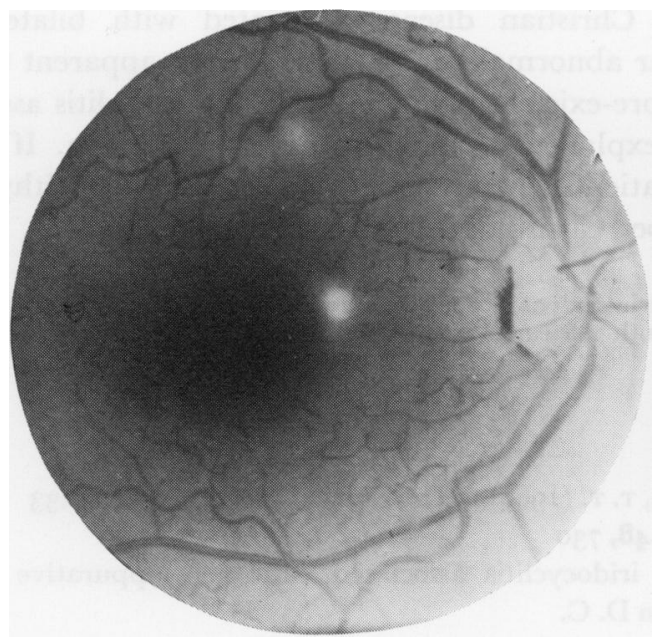

FIG. 3 Right eye after 2 weeks

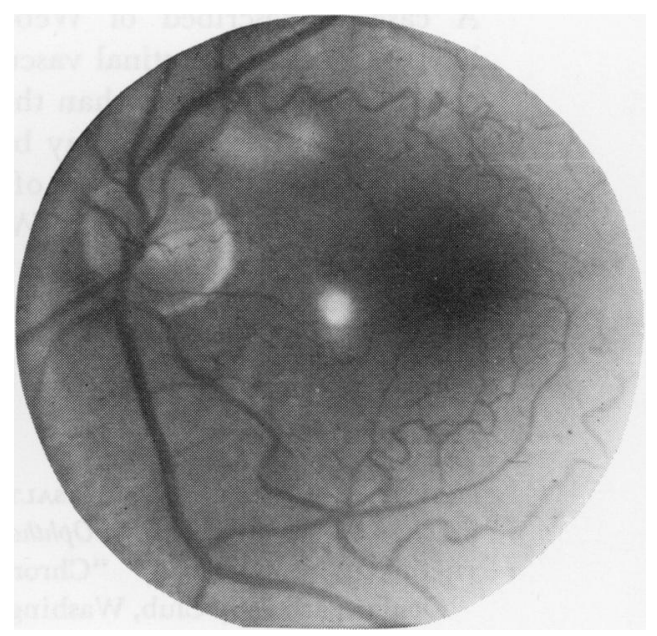

FIG. 4 Left eye after 2 weeks

At the time of the occurrence of the ocular lesions, the patient, although having nodules on the under surfaces of the thighs, was receiving no therapy. The disease process had been present for 7 years, and when first diagnosed she had been treated with betamethasone $0.5 \mathrm{mg}$. twice daily, and was kept on systemic steroids for 2 months. The tenderness of the nodules improved on this therapy and steroids were discontinued. After a recurrence of the painful nodules on the under surfaces of the thighs, a course of thyroxin was tried but with little effect. She was then given phenylbutazone Ioo mg. four times a day and there was improvement. During this time she also received a 2-week course of terramycin 2,00o mg./day. Up to the time when the ocular lesions appeared, she had been 
having intermittent attacks of painful nodules on the under surfaces of the thighs and these had been treated mainly with phenylbutazone $\mathrm{I} 00 \mathrm{mg}$. four times a day. Short periods of steroid therapy had also been given when the crops of painful nodules recurred.

\section{Discussion}

The Weber Christian syndrome is also known as recurrent nodular non-suppurative panniculitis. Klien (1959) gave a comprehensive description of the disease, which occurs most often in middle-aged and elderly women, being characterized by malaise and fever and subcutaneous nodules on the trunk and extremities. The nodules represent local areas of fat necrosis. The necrotic area is invaded at first by acute inflammatory cells and later by lymphocytes and monocytes, and ultimately the nodule is replaced by fibrous tissue. The sclerosis is accompanied by a mild vasculitis. When uveitis occurs it tends to be recurrent.

Klien (1959) suggested that the occurrence of uveitis and panniculitis might indicate the patient's sensitivity and foreshadow more widespread tissue responses. The involvement of the retinal vasculature, as in the above patient, is therefore not unexpected, but simultaneous macular haemorrhages are somewhat unique. This was the first ocular manifestation in our patient, so that retinal haemorrhages or macular involvement may recur. Weber Christian disease thus appears to be one in which the eye may become involved in a diverse manner, and other ocular manifestations may be observed in the future.

\section{Summary}

A case is described of Weber Christian disease associated with bilateral macular haemorrhages and retinal vascular abnormalities. There was no apparent cause for the ocular pathology other than the pre-existent panniculitis. The vasculitis associated with Weber Christian disease may be explained as a hypersensitivity reaction. If pathological changes are found in the eyes of patients who are otherwise apparently healthy, an enquiry into the possible existence of Weber Christian disease should be made.

My thanks are due to the Medical Superintendent of the Johannesburg General Hospital for permission to publish this case report, and to the South African Medical Research Council and the University of the Witwatersrand for financial assistance.

\section{References}

Frayer, W. C., Wise, R. T., and tsaltas, t. t. (1968) Trans. Amer. ophthal. Soc., 66, 233

KLIEn, B. A. (1959) Amer. J. Ophthal., 48, $73^{\circ}$

THEOBALD, G. D. (I953) "Chronic iridocyclitis associated with non-suppurative panniculitis." Ocular Pathology Club, Washington D. C. 